\title{
Phylogenetic analysis of phytochrome A gene from Lablab purpureus (L.) Sweet
}

\author{
Stuti Krishna ${ }^{1}$, Kaushal Modha ${ }^{1 *} \mathbb{D}$, Vipulkumar Parekh², Ritesh Patel ${ }^{1}$ and Digvijay Chauhan ${ }^{3}$
}

\begin{abstract}
Background: Phytochromes are the best characterized photoreceptors that perceive Red (R)/Far-Red (FR) signals and mediate key developmental responses in plants. It is well established that photoperiodic control of flowering is regulated by PHY A (phytochrome A) gene. So far, the members of PHY A gene family remains unexplored in Lablab purpureus, and therefore, their functions are still not deciphered. PHYA3 is the homologue of phytochrome $A$ and known to be involved in dominant suppression of flowering under long day conditions by downregulating florigens in Glycine max. The present study is the first effort to identify and characterize any photoreceptor gene (PHYA3, in this study) in Lablab purpureus and decipher its phylogeny with related legumes.

Results: PHYA3 was amplified in Lablab purpureus CV GNIB-21 (photo-insensitive and determinate) by utilizing primers designed from GmPHYA3 locus of Glycine max. This study was successful in partially characterizing PHYA3 in Lablab purpureus (LprPHYA3) which is $2 \mathrm{~kb}$ longer and belongs to exon 1 region of PHYA3 gene. Phylogenetic analysis of the nucleotide and protein sequences of PHYA genes through MEGA X delineated the conservation and evolution of Lablab purpureus PHYA3 (LprPHYA3) probably from PHYA genes of Vigna unguiculata, Glycine max and Vigna angularis. A conserved basic helix-loop-helix motif bHLH69 was predicted having DNA binding property. Domain analysis of GMPHYA protein and predicted partial protein sequence corresponding to exon-1 of LprPHYA3 revealed the presence of conserved domains (GAF and PAS domains) in Lablab purpureus similar to Glycine max.

Conclusion: Partial characterization of LprPHYA3 would facilitate the identification of complete gene in Lablab purpureus utilizing sequence information from phylogenetically related species of Fabaceae. This would allow screening of allelic variants for LPrPHYA3 locus and their role in photoperiod responsive flowering. The present study could aid in modulating photoperiod responsive flowering in Lablab purpureus and other related legumes in near future through genome editing.
\end{abstract}

Keywords: Phytochrome, PHYA3, GAF, PAS, Phylogenetic analysis

\section{Background}

Photoperiod sensitivity is an important trait as it enables crops to adapt to different latitudes. Short-day (SD) crops like Oryza sativa, Lablab purpureus, Phaseolus vulgaris and Glycine max requires photoperiod insensitivity to adapt to a high latitudinal environment [1]. Photoperiod

\footnotetext{
*Correspondence: kgmodha@nau.in

1 Department of Genetics and Plant Breeding, N. M. College

of Agriculture, Navsari Agricultural University, Navsari, Gujarat 396 450,

India

Full list of author information is available at the end of the article
}

is governed by phytochrome genes which play critical role in responding to the light quantity, quality and periodicity in plants and make communication between different biochemicals signaling pathway for growth and development of crops [2]. Phytochromes are Red (R)/ Far-Red (FR) light receptors involving interconversion of inactive $\mathrm{R}(\mathrm{Pr}$ ) to active FR (Pfr) form by red light absorption which triggers its transfer to the nucleus and thus guides gene expression [3]. Phytochrome is the best characterized photoreceptor and its apoproteins are encoded by small multigene families. In recent decade, progress has been made in characterizing the number, molecular 
properties and biological activity of the photoreceptors that comprise a plant R/FR detecting framework [2].

Legumes have been documented with vast genetic variation for flowering and photoperiod has been quite involved in governing growth habit, flowering and maturity in these crops [4]. Legumes have been the focus of presently expanding genomics research and the availability of vast genomic resources and established synteny within the Fabaceae family has enabled the identification of candidate genes for flowering in other related species and exploring their molecular physiologies involved in downstream processes [5]. The fundamental genes governing flowering pathway in legumes are conserved from model plant Arabidopsis that contains five $P H Y$ (phytochrome) genes (PHYA, PHYB, PHYC, PHYD and $P H Y E)$ and each of these gene have crucial role $[6,7]$. Different maturity loci, i.e. E-series ( $E 1$ to $E 8$ ) represent a range of allelic composition and each $E$ locus affects flowering time and maturity in Glycine max [8-14]. Photoperiod sensitivity under different light conditions was found to be related to $E 1, E 3, E 4$ and $E 7$ [15]. E3 and $E 4$ encode GmPhyA3 and GmPhyA2, respectively, which are homologues of photoreceptor phytochrome A [16]. GmPHYA3 and GmPHYA2 along with GmPHYA1, which is encoded by a homoeologous copy of $E 4$, redundantly or complementarily function in floral induction and deetiolation responses under various light conditions [17]. $E 3$ and E4 coordinates flowering responses to long-day (LD) conditions with different Red-to-Far-Red (R:FR) quantum ratios. $E 3$ responds to light with high R:FR ratio; plants homozygous for the recessive $e 3$ allele can initiate flowering under the LD conditions generated by fluorescent lamps with a high R:FR ratio [18]. Photoperiod sensitivity and determinacy played important role in evolution of Phaseolus vulgaris [19]. GmFT2a and GmFT5a, two homologues of $F T$ (Flowering locus T), are reported to induce photoperiodic flowering in Glycine max [20] and they are regulated by E1, E3 (PHYA3) and E4 genes under different photoperiod conditions to induce or repress flowering [15]. Photoperiodic response of $P H Y A 3$ under short day and long day conditions affects growth habit by inducing other genes that activate florigens and guides floral initiation at shoot apex [3].

The research on photoperiod responsive flowering is mainly anchored to few major pulse legumes viz., Glycine max, Pisum sativum, Phaseolus vulgaris, Cajanus cajan and vegetable legumes endured despite being a promising nutritional source. Indian bean [Lablab purpureus (L.) Sweet] is one such underexploited legume with wide range of uses as vegetable, forage, cover crop, split pulse, fodder and medicinal [21]. The molecular information regarding this crop is scarce; nevertheless, it has the potential to become a model pulse crop in genomics era owing to its immense genetic diversity and adaptability [22]. Most landraces of Lablab purpureus are photoperiod sensitive and indeterminate which flower only during short days, while few improved varieties with determinate growth and photoperiod insensitivity are available which flowers within 40 to 50 days across the year. Photoperiod responsive flowering along with growth habit might have played crucial role in domestication and evolution of this crop. Dominant nature of photoperiod sensitivity, indeterminate growth habit and purple flower was reported along with coupling phase of linkage between photoperiod insensitive flowering and determinate growth habit in Lablab purpureus [23]. Molecular tagging of photoperiod responsive flowering in Lablab purpureus discerned that photoperiod insensitive flowering and determinate growth habit is linked and they might be governed by recessive alleles of GmPHYA3 and $D t$ homologs, respectively [24]. Most recently, allelic characterization of TFL (Terminal Flowering locus) governing growth habit has been reported along with involvement of splice site single nucleotide polymorphism (SNP) for growth habit differences in Lablab purpureus [25]. The role of PHYA3 in photoperiod responsive flowering is already reported in Glycine max and Phaseolus vulgaris, and mutations in E3/PHYA3 conferred photoperiod insensitive and early flowering $[3,26]$. Lack of genome sequence data or any molecular information regarding $P H Y A 3$ gene or any marker tightly linked to it in Lablab purpureus has limited the molecular characterization of photoperiod responsive flowering in this crop. Looking to possible role of $P H Y A 3$ in photoperiod responsive flowering and lack of genome sequence information, the present work is focused on characterization of this gene in Lablab purpureus using candidate gene approach and phylogenetic analysis of legume phytochromes.

\section{Materials and methods \\ Primer designing}

The GmPHYA3 sequence from Glycine max [3] was obtained in FASTA format from NCBI (National Centre for Biotechnology Information) GenBank database (https://www.ncbi.nlm.nih.gov/genbank/). GmPHYA3 is $9.2 \mathrm{~kb}$ longer, exon 1 was divided into three frames and primers for each frame were designed using web-based Primer BLAST (Basic Local Alignment Search Tool) from NCBI (Table 1). These primers were utilized for amplification of PHYA3 in Lablab purpureus cv GNIB-21 which is determinate and photoperiod insensitive.

\section{Amplification and sequencing}

Genomic DNA was isolated from young fresh trifoliate leaves of GNIB-21 using CTAB method [27]. The DNA quality and quantity were assessed using gel 
Table 1 Primers used for partial amplification LprPHYA3 locus

\begin{tabular}{llll}
\hline Frame & Primer & Sequence & $\begin{array}{l}\text { Amplicon } \\
\text { length } \\
\text { (bp) }\end{array}$ \\
\hline Frame-1 & $93 \mathrm{~F}$ & 5'TGCATCAGATAACAGTGGAAGA3' & 951 \\
& $94 \mathrm{R}$ & 5'TTGTAGGATTGCAGGGCTCC3' $^{\prime}$ & \\
Frame-2 & $115 \mathrm{~F}$ & 5'ATTTTGAGCCGGTCAAGCCT3' $^{\prime}$ & 987 \\
& $116 \mathrm{R}$ & 5'CAGCTGCCATTCCACATGC3' $^{\prime}$ & \\
Frame-3 & $133 \mathrm{~F}$ & 5'TTGTCTGATGCAGGCTTCCC3' & 964 \\
& $134 \mathrm{R}$ & 5'CCAAGCTGATGGGACCAGAA3' & \\
\hline
\end{tabular}

$F$ and $R$ represent forward and reverse primers, respectively

electrophoresis and spectrophotometer (Nanodrop 2000, Thermo Fisher, USA). Both the stock and diluted DNA preparations were stored at $-50^{\circ} \mathrm{C}$ until use. The target frames were amplified in GNIB-21 through Polymerase Chain Reaction (PCR) with Taq DNA polymerase (TaKaRa, Clontech, Japan). PCR mixture prepared in $200 \mu \mathrm{l}$ contained approximately $100 \mathrm{ng}$ genomic DNA, $200 \mu \mathrm{M}$ of dNTPs, 10 pmol of forward and reverse primers, standard Taq buffer $\left(\mathrm{Mg}^{2+}\right.$ plus) and 1 unit of Taq DNA polymerase in total volume of $25 \mu \mathrm{l}$ reaction. The PCR cycle involved initial denaturation of $95^{\circ} \mathrm{C}$ for 7 min followed by 35 cycles of $94^{\circ} \mathrm{C}(30 \mathrm{~s}), 51-55^{\circ} \mathrm{C}(45$ s) and $72^{\circ} \mathrm{C}(1 \mathrm{~min})$ and a final extension of $10 \mathrm{~min}$ at $72^{\circ} \mathrm{C}$. Amplicons giving the single discrete band when resolved on $1.5 \%$ agarose gel electrophoresis with the expected product size were purified using column purification with SLS PCR Clean-up Kit (Saffron Life science, Surat, India). Sanger sequencing reaction of purified PCR amplicon was carried out with specific primers using BDT v3.1 Cycle sequencing kit on ABI 3730xl Genetic Analyzer (Applied Biosystems). Bidirectional sequence data obtained from each amplicon were processed using BioEdit [28]. The bidirectional sequence information obtained were processed by merging the sequences from three frames and overlapping sequences were identified in both directions to construct consensus sequence.

\section{Sequence retrieval, alignment and phylogenetic analysis}

The processed $\operatorname{LprPHYA3}$ sequence was used as query for BLASTn at https://blast.ncbi.nlm.nih.gov/Blast. cgi for finding homologous sequences with reference to NCBI (National Center for Biotechnology Information) nucleotide database [29]. Sequences showing matches with LprPHYA3 from Fabaceae family were retrieved from NCBI nucleotide database. MEGA X (Molecular Evolution Genetics Analysis) software [30] was used to align PHYA nucleotide sequences of 16 species from Fabaceae family in addition to Arabidopsis thaliana using the CLUSTAL W alignment algorithm [31]. All the alignment settings were employed at default values; 2160 and 2051 positions with and without gaps were obtained, respectively. The nucleotide substitutions selected with complete deletion of gaps or missing data were used to analyse sequences. The phylogenetic tree was inferred using Maximum Likelihood method based on TamuraNei Model [32]. The initial tree was inferred with default setting using Neighbour Joining method and NearestNeighbour Interchange was used as ML heuristic search method. The reconstructions of phylogenetic trees were conducted using Maximum Likelihood Method. Bootstraps with 1000 replicates for Poisson correction model were performed to assess node support [33]. The bestscoring ML tree was searched simultaneously to represent the evolutionary history of the 20 specimens tested.

\section{Prediction of conserved motifs}

The detection of conserved motifs in DNA sequence of phytochrome gene was performed using online tool MEME (Multiple Em for Motif Elicitation) (https:// meme-suite.org/meme/) [34]. This online web-based analysis was performed with minimum and maximum motif width of 6 and 12 residues, respectively, which were used to identify probable motifs, keeping the rest of the parameters at default. The MEME output in HTML showed the motifs as local multiple alignments of the input sequences, as well as in several other formats. MEME HTML output were allowed one or all of the motifs to be forwarded for additional investigation. The results of the MEME analysis were applied to TOMTOM (http://memesuite.org/tools/tomtom), which is the online software performing comparison of given motifs with available databases. The output generated from TOMTOM include sequence-logo graphics on behalf of the alignment of two motifs with $\mathrm{p}$ and $\mathrm{q}$ value (a measure of false discovery rate) of the match [35].

\section{Exon prediction, protein prediction and functional analysis} The identified partial sequence of LprPHYA3 was subjected to exon prediction using Eukaryotic GeneMark. $\mathrm{hmm}$ version 3.54 [36]. The nucleotide sequence of exon-1 obtained in such a way was translated to protein sequence using standard codon table. This amino acid sequence obtained was further compared with GmPHYA3 sequence. The protein sequence of exon 1 from Lablab purpureus GNIB-21 was used to perform BLASTp in NCBI GenBank database and the sequences showing homology were further used to create multiple alignment using the CLUSTAL W algorithm [29, 31]. Phylogenetic analysis with amino acid sequence of $P H Y A$ in 21 different plant species was also performed using JTT model in MEGA X [30, 37]. The online web-based functional analysis tool SMART-EMBL (Simple Modular 


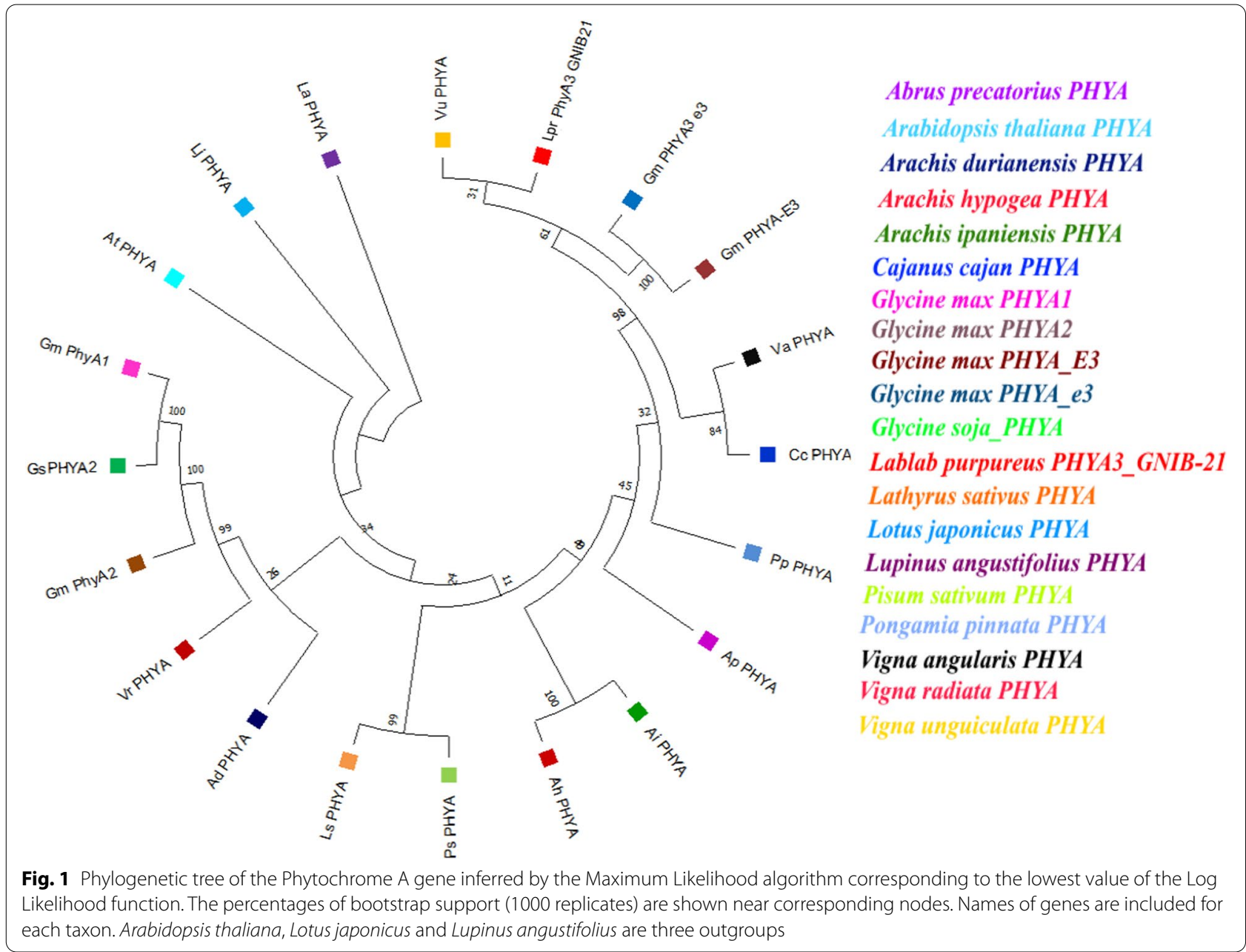

Architecture Research Tool) (http://smart.embl-heide lberg.de/) Version 9 was used for predicting domains in both GmPHYA3 and LprPHYA3 protein sequences [38].

\section{Results}

\section{LprPHYA3 characterization and phylogenetic analysis}

In the present study, Lablab purpureus cv GNIB-21, which is determinate and photo-insensitive, was used for characterizing PHYA3 gene. The sequencing data after processing and analysis revealed the successful characterization of the exon-1 of PHYA3 gene in Indian bean (LprPHYA3- Lablab purpureus PHYA3) for the first time in the world. BLASTn analysis of nucleotide sequence LprPHYA3 indicated highest identity with Vigna unguiculata (95.76\%) followed by Vigna angularis (95.67\%) and Glycine max (90.90\%), all with $E$-value close to zero.

Phylogenetic tree depicts formation of different clades on the basis of evolutionary changes between the sequences. LprPHYA3 nucleotide sequence was compared with the nucleotide sequences of phytochrome genes of other plant species, most of which, belonged to the Fabaceae family. Phylogenetic analysis showed that this phytochrome A gene evolved from common ancestry root but diverged into different clades during the course of evolution (Fig. 1). The PHYA sequences from Lablab purpureus, Vigna unguiculata and Glycine max form independent clade and are closest to Vigna angularis and Cajanus cajan. This indicates that LprPHYA3 has maximum closeness to Vigna unguiculata, Glycine max, Cajanus cajan and Vigna angularis PHYAs suggesting the evolutionary closeness of the gene in these species. The three outgroups depicted in the tree are Lupinus angustifolius, Lotus japonicus and Arabidopsis thaliana.

Realizing the importance of both DNA and protein sequence alignment in phylogenies, the protein sequence predicted from the exon 1 of $\operatorname{LprPHYA3}$ gene was also studied along with the other sequences to delineate the amino acid changes in the sequence during the course of evolution. Alignment of the amino acid sequences from PHYA homologs was done by CLUSTAL W alignment 


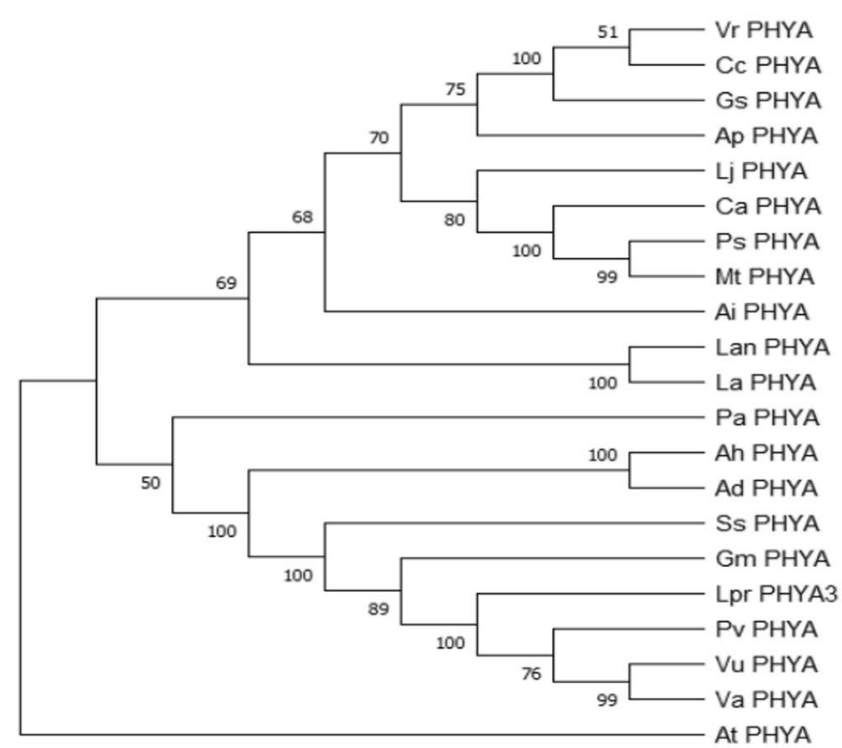

- Vigna radiata $P H Y A$

- Cajanus cajan PHYA

- Glycine soja PHYA

- Abrus precatorious PHYA

- Lotusjaponicus PHYA

- Cicer arientium PHYA

- Pisun sativun PHYA

- Medicago truculata PHYA

- Arachisipaniensis PHYA

- Lupinus angustifolius PHYA

- Lupinus albus PHYA

- Prosopis alba PHYA

- Arachis lypogea PHYA

- Arachis durianesis PHYA

- Spatholobus suberectus PHYA

- Glycine max PHYA

- Lablab purpureus PHYA3

- Phaseolus vulgaris PHYA

- Vigna unguiculata PHYA

- Vigna angularis PHYA

- Arabidopsis thaliana PHYA

Fig. 2 Phylogenetic tree of amino acid sequences of phytochrome A by Maximum Likelihood algorithm showing evolutionary lineage using MEGA $X$ software. The bootstrap consensus tree is inferred from 1000 replicates; with the confidence values shown next to the branches, Arabidopsis thaliana is an outgroup

algorithm and phylogenetic tree for protein sequences was constructed (Fig. 2). The protein alignment also depicts the most probable evolution of LprPHYA3 from common ancestral PHYA gene of Glycine max, Phaseolus vulgaris, Vigna unguiculata and Vigna angularis. This tree depicts Arabidopsis thaliana as an outgroup.

\section{Determination of common motifs in PHYA sequence}

Identification of conserved motifs in the $P H Y A$ was performed by comparing DNA sequences from these 20 PHY A sequences from different species. The analysis resulted in three conserved sites present in pool of studied sequence. The best result was a 12-nt-long motif GTGCAAAGCATG which was found in all 20 analysed sequence (Table 2). The $E$-value for best match discussed here was $3.4 \times 10^{-45}$. Subsequently, the motif was compared using TOMTOM with a database of Arabidopsis thaliana DAP motifs which resulted in identification of bHLH69 basic helix-loop-helix motif containing transcription factor ( $p$ value $1.16 \times 10^{-4}$ and $E$ value $1.01 \times 10^{-1}$ ) (Fig. 3).

\section{Domain analysis of GmPHYA3 and LPrPHYA3}

Domain analysis of GmPHYA3 sequence retrieved from NCBI GenBank database after performing BLASTp search with LprPHYA3 that encodes a protein with 1130 amino acids was carried out using EMBL-SMART platform. This protein displayed normal features of Phytochrome A with five domains viz., GAF (cGMP-specific phosphodiesterase-adenylyl cyclase-FhlA), two PAS
(period-ARNT-single-minded) domains and two Histidine kinase-related domains (HKRD) viz., HisKA and HATPase_c as depicted in Fig 4a. The amino acid sequence of $\operatorname{LprPHYA3}$ showed two domains, i.e. GAF sand PAS (Fig 4b). This finding is in congruity with the domain analysis from GmPHYA3.

\section{Discussion}

The deciphering of putative molecular pathways in legumes involving different phytochrome genes and their involvement in governing flowering time will pave way for future research. Various genetic models for this regulatory framework of photoperiod-based flowering with different known loci is available for Glycine max. It is studied in depth for photoperiod control of flowering and different $E$ loci have been reported, out of which, $E 3$ (GmPHYA3) is considered a strong candidate for FT3 and mutations in GmPHYA3 results in early flowering [3]. Different mutations of $E 3$ have been reported in Glycine max and Phaseolus vulgaris that confer significantly early flowering and photoperiod insensitivity [3, 26]. Additionally, naturally occurring E3 mutants depicted a large insertion in fourth intron and one SNP for nonsynonymous amino acid substitution in third exon. Naturally occurring $e 3$ allele carries a large deletion spanning exon 4 , whereas an induced mutant $e 3$ allele has sustained a 40-bp deletion and frameshift in the middle of exon 1 [3]. Flowering time is a very important trait governing several other correlated functions and molecular identification of flowering networks with these $E$ loci can 
Table 2 Predicted conserved motifs in 20 sequences of Phytochrome A gene

\begin{tabular}{|c|c|c|c|c|c|c|}
\hline Sequence & Length & Start & $P$-value & Sites & & \\
\hline Ad_PHYA & 2054 & 1598 & $5.50 \times 10^{-8}$ & AGATGGGGTG & GTGCAAAGCATG & ACCCTGGTGA \\
\hline Ah_PHYA & 2057 & 884 & $5.50 \times 10^{-8}$ & GTTGATTGTC & GTGCAAAGCATG & TGAAGGTGCT \\
\hline Ai_PHYA & 2057 & 884 & $5.50 \times 10^{-8}$ & GTTGATTGTC & GTGCAAAGCATG & TGAAGGTGCT \\
\hline$A p \_P H Y A$ & 2073 & 896 & $5.50 \times 10^{-8}$ & GTTGATTGTC & GTGCAAAGCATG & TGAAAGTCCT \\
\hline At_PHYA & 2057 & 1601 & $1.42 \times 10^{-7}$ & AGATGGGGAG & GTGCAAAGCATG & ATCCAGATGA \\
\hline CC_PHYA & 2076 & 896 & $5.50 \times 10^{-8}$ & GTTGATTGTC & GTGCAAAGCATG & TTAAGGTGCT \\
\hline Gm_PHYA1 & 2077 & 1589 & $5.50 \times 10^{-8}$ & CGATGGGGTG & GTGCAAAGCATG & AGGCTGGAGA \\
\hline Gm_PHYAZ & 2072 & 1589 & $5.50 \times 10^{-8}$ & CGATGGGGTG & GTGCAAAGCATG & AGGCTGGAGA \\
\hline Gm_PHYA3_E3 & 2077 & 899 & $5.50 \times 10^{-8}$ & GTTGATTGTT & GTGCAAAGCATG & TGAATGTGCT \\
\hline Gm_PHYA3_e3 & 2077 & 899 & $5.50 \times 10^{-8}$ & GTTGATTGTT & GTGCAAAGCATG & TGAATGTGCT \\
\hline Gs_PHYA2 & 2056 & 1595 & $5.50 \times 10^{-8}$ & CGATGGGGTG & GTGCAAAGCATG & AAGCTGGAGA \\
\hline La_PHYA & 2063 & 454 & $5.50 \times 10^{-8}$ & CGCTGGGGTG & GTGCAAAGCATG & AACCTGGTGA \\
\hline Lj_PHYA & 2059 & 1598 & $5.50 \times 10^{-8}$ & CGATGGGGTG & GTGCAAAGCATG & AACCTGGAGA \\
\hline Lpr_PHYA3 & 2077 & 902 & $5.50 \times 10^{-8}$ & GTTGATTGTC & GTGCAAAGCATG & TGAAGGTGCT \\
\hline LS_PHYA & 2059 & 1598 & $5.50 \times 10^{-8}$ & CGATGGGGTG & GTGCAAAGCATG & AACCGGGCGA \\
\hline Pp_PHYA & 474 & 26 & $3.18 \times 10^{-7}$ & GTTGATTGTC & GCGCAAAGCATC & TGAAGGTTCT \\
\hline PS_PHYA & 2056 & 1598 & $5.50 \times 10^{-8}$ & CGATGGGGTG & GTGCAAAGCATG & AACCGGGCGA \\
\hline Va_PHYA & 2079 & 902 & $5.50 \times 10^{-8}$ & GTTGATTGTC & GTGCAAAGCATG & TGAAGGTGCT \\
\hline Vr_PHYA & 2051 & 1595 & $5.50 \times 10^{-8}$ & CGATGGGGTG & GTGCAAAGCATG & AAGCTGGAGA \\
\hline VU_PHYA & 2079 & 902 & $5.50 \times 10^{-8}$ & GTTGATTGTC & GTGCAAAGCATG & TGAAGGTTCT \\
\hline
\end{tabular}

The column representing sequences are phytochrome A (PHYA) nucleotide sequences from 20 species. Ad_PHYA Arachis durianesis, Ah_PHYA Arachis hypogea, Ai_PHYA Arachis ipaniensis, Ap_PHYA Abrus precatorious, At_PHYA Arabidopsis thaliana, Cc_PHYA Cajanus cajan, Gm_PHYA1, Gm_PHYA2, Gm_PHYA3_E3, Gm_PHYA3 e3 Glycine max PHY A homologs, Gs_PHYA2 Glycine soja, La_PHYA Lupinus angustifolius, Lj_PHYA Lotus japonicus, Lpr_PHYA3 Lablab purpureus, Ls_PHYA_Lathyrus sativus, Pp_ PHYA Pongamia pinnata, Ps_PHYA Pisum sativum, Va_PHYA Vigna angularis, Vr_PHYA Vigna radiata, Vu_PHYA Vigna unguiculata. The following columns present length of DNA sequence, start position, random letters probability and the sequence of the conserved motif

be used for more efficient breeding strategies [39]. These pathways have been more or less conserved in related legume species involving numerous loci with known or unknown functions. On the basis of available information on related genes and pathway undermining photoperiodic flowering in legumes, a theoretical model for photoperiod dependent flowering pathway is proposed (Fig. 5). In Lablab purpureus, photoperiodic response of flowering may also be governed by circadian clock as it is reported in Glycine max. CONSTANS (CO) is a circadian-regulated gene and acts as prime regulator of this pathway. It activates the expression of florigen gene $F T$ by coordinating light and clock input to leaves. The different genes, their responses to photoperiod and their putative roles mediating this process have been proposed. It is speculated that under SD condition (low R:FR), E3 and $E 4$ are repressed due to lack of exorbitant light condition and thus $E 1$ is also suppressed which thus has no effect on FT2 $a$ and FT5 a genes leading to early flowering phenotype. Under enriched light condition, i.e. LD condition PHYA3 (E3) and PHYA2 (E4) are expressed activating the expression of $E 1$ which eventually leads to $F T 2 a / F T 5 a$ downregulation, flowering repression, indeterminate growth (if TFL1 is present) and delayed maturity. The presence of TFL1 and/or $t f l$ convoys indeterminate and determinate growth habit, respectively $[1,15]$. Dominant TFL allele suppresses development of floral architecture at shoot apex in indeterminate types; racemes emerge from axillary bud only upon short day conditions where $F T$ s, expressed due to favourable photoperiod condition, might be able to nullify the effect of TFL in competitive manner. Determinate growth habit results from non-functional allele $(t f l)$ of $T F L$ which is unable to suppress flowering in shoot apex and results into determinate growth habit and photoperiod insensitive flowering. This is unveiled by the presence of splice site SNP at third exon in Lprtfl which renders non-functional protein and is responsible for determinate growth habit and photoinsensitive flowering in Lablab purpureus cv GNIB-21 [25]. A non-synonymous SNP in exon 4 of TFL1 locus in cowpea also resulted in determinate growth habit [40]. However, photoperiod responsive expression of growth habit cannot be ignored [41].

Legumes have always been of persistent interest due to vast genetic variation for photoperiod responses mediating crucial traits like growth habit, flowering and maturity. A summary of different phytochrome $A$ genes 

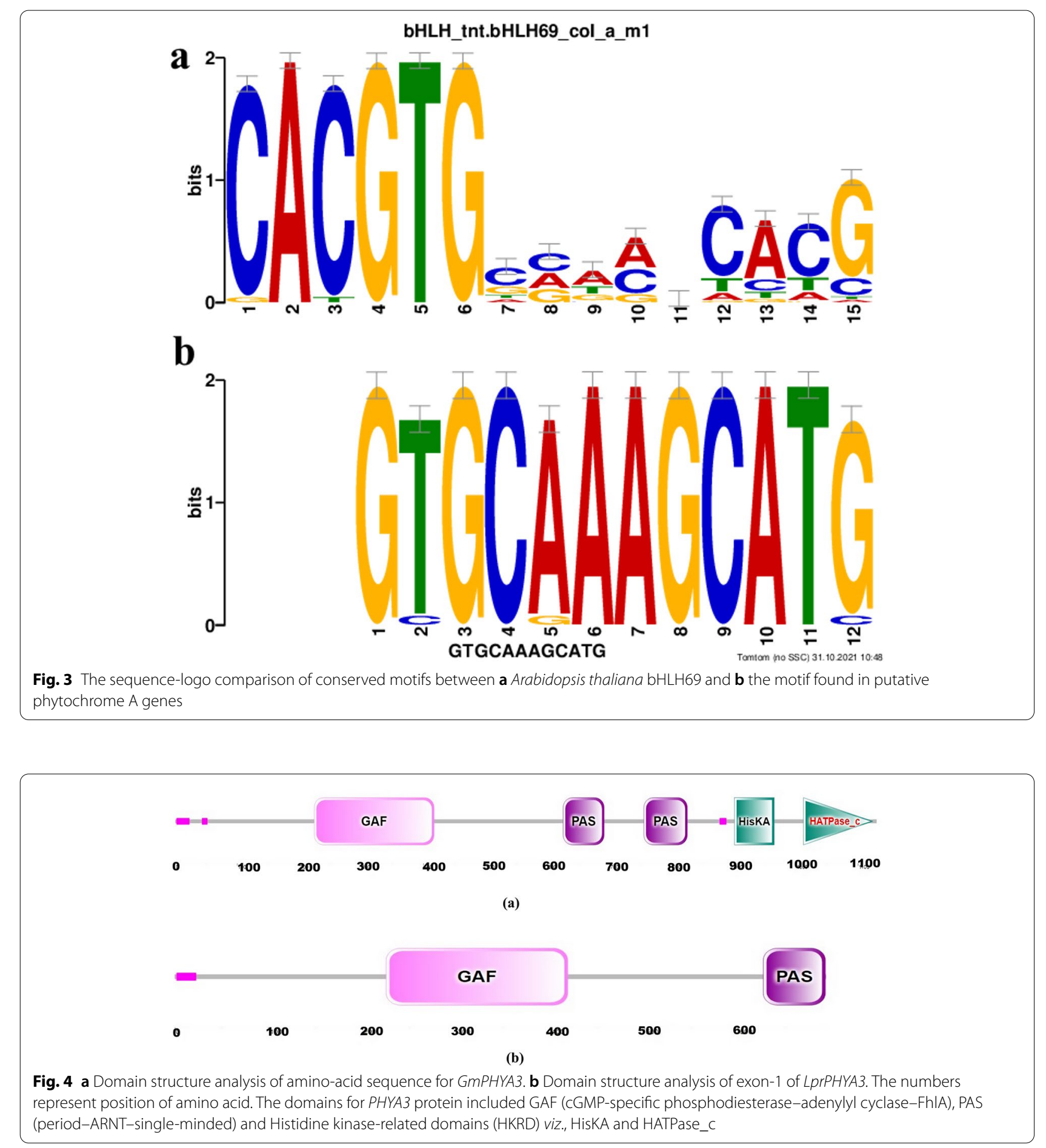

in some legumes along with their putative role is represented (Table 3).

Seeking the importance of GmPHYA3 (or E3) in photoperiod adaptation of short-day legumes like Glycine max and Phaseolus vulgaris, it becomes quite important to understand its role in Lablab purpureus which is also a short-day crop. Due to the lack of genome sequence database, there is very scanty information available about Lablab purpureus at molecular level. The present study was successful in characterizing exon-1 in LprPHYA3 by utilizing GmPHYA3 sequence as reference. Comparative gene mapping has been quite useful in deducing genomic 


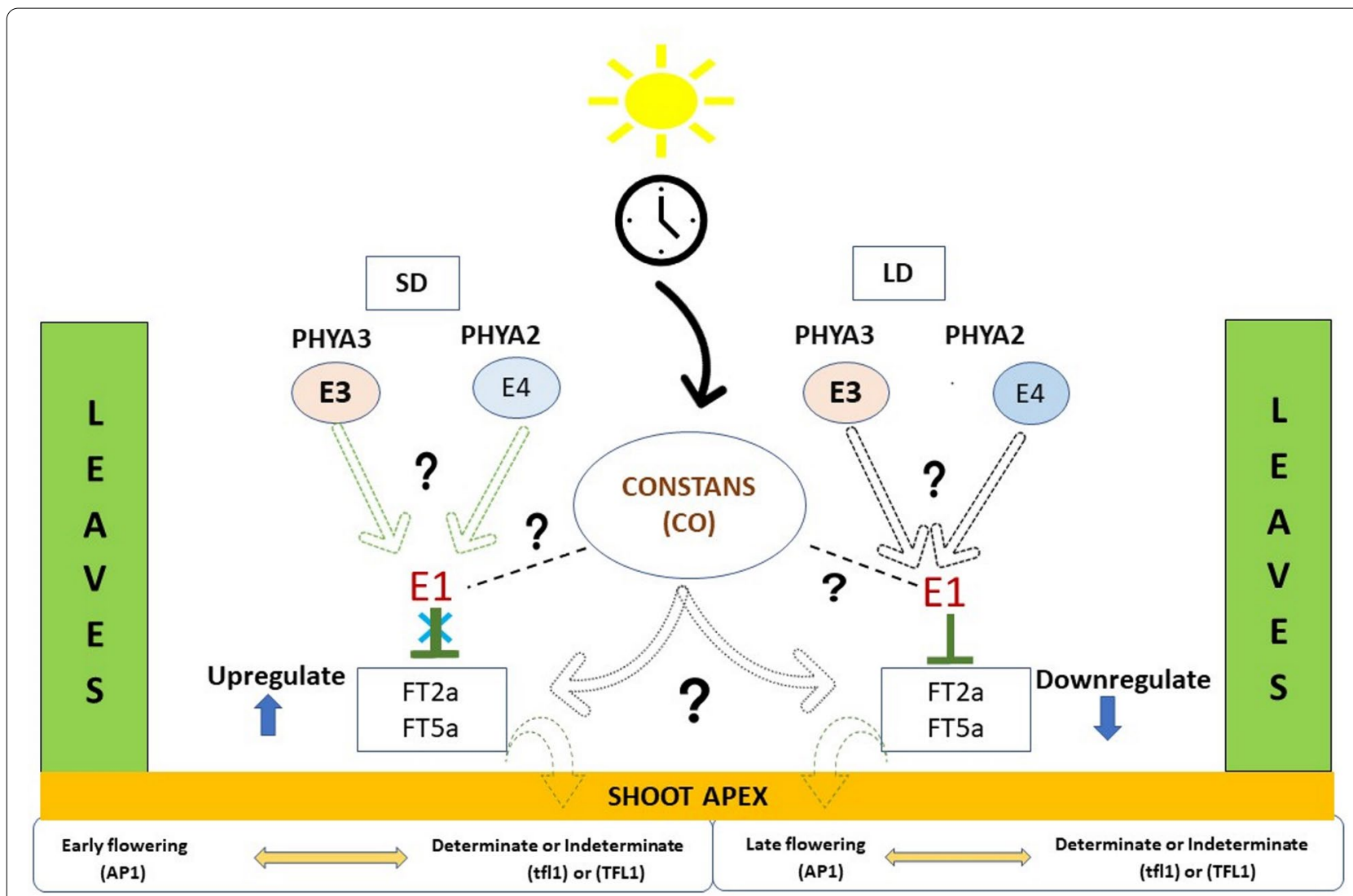

Fig. 5 A theoretical model for photoperiod responsive flowering and growth habit mediating plant architecture in response to short day (SD) and long day (LD) conditions based on genes reported in Lablab purpureus, Glycine max and related legumes. Light arrows in SD indicate repressed $E 3$ and E4 during short days. Question marks indicate the unknown genes involved in mediating the process. Genes E4, E1, FT2a and FT5a are yet to be identified in Lablab purpureus. PHYA3 and PHYA2 are homologs of phytochrome A. E1, E3 and E4 are maturity loci governing photoperiodic regulation in Glycine max. FT2 $a$ and FT5 a are Flowering locus T homologs reported as florigens in many legumes and induce flowering

structure from related plant species due to conservation of genetic content, gene order and function $[25,46]$. This marks the significance of phylogenetic analysis to depict evolutionary closeness of different plant species. DNAbased phylogenies have been dominated in recent years mainly because it yields more phylogenetic information than protein. Since non-synonymous mutations do not affect amino acid sequence but do alter nucleotide sequences of a pair of homologous genes. Both DNA- and amino acid sequence-based phylogenies have been conducted in present study for validation of results obtained by phylogenetic analysis. DNA sequence phylogenies provide opportunity to the researcher for examination of both coding and non-coding regions of gene. However, the use of protein sequences in establishing evolutionary relationships cannot be ignored as amino acid sequences are more conserved than DNA sequences. The degeneracy in genetic code and difference in codon usage in different species makes it less accepted. Protein sequence gives more sensitive sequence alignment as DNA has only 4 characters, while protein has 20 . The translation of DNA to protein gives a higher signal-to-noise ratio and thus sharpens up the analysis making it better for phylogenetic studies [47]. PHYA3 gene has most probably been evolved from common ancestral PHYA gene of these species, as depicted from the tree. Previous studies have also shown closeness of Lablab purpureus to Vigna unguiculata and their predictable evolution from Glycine max in Phaseoleae clade [48]. This study has delineated the conservation of PHYA3 among the phaseoleae clade legumes indicating evolutionary closeness with Vigna unguiculata, Vigna angularis and Glycine max employing both DNA and protein sequences for phylogenetic analysis (Figs. 1 and 2). The evolutionary relationship of Lablab purpureus to Glycine max, Vigna unguiculata and Phaseolus vulgaris among the Phaseoleae clade has been reported earlier [48-50]. This will aid in isolating allelic variants of PHYA3 from Lablab purpureus by utilizing model plants like Glycine max and Phaseolus vulgaris. Characterization of the full gene could pioneer the 
Table 3 Summary of identified Phytochrome A genes in different crop legumes

\begin{tabular}{|c|c|c|c|}
\hline Gene & Legume species & Function/role & Reference \\
\hline PHYA3 E3 & Glycine max & R/FR photoreceptor; represses FT; delays flowering in LD & [3] \\
\hline PHYAЗ e3 & Glycine max & Promotes flowering in LD & {$[42]$} \\
\hline PHYA2 E4 & Glycine max & R/FR photoreceptor; represses FT; delays flowering in LD & [17] \\
\hline PHYAT & Glycine max & Unknown & [17] \\
\hline PHYA2 E4 & Glycine soja & Inhibits flowering & [42] \\
\hline PHYA & Pisum sativum & R/FR photoreceptor & [43] \\
\hline PHYA3 & Phaseolus vulgaris & R/FR photoreceptor; represses FT; delays flowering in LD & {$[26]$} \\
\hline PHYA and PHYB & Arachis hypogea & Gynophore enlargement & [44] \\
\hline PHYA & Vigna radiata & Control FT & [45] \\
\hline
\end{tabular}

extensive photoperiodic flowering control mechanism in Lablab purpureus.

Phytochromes mediates light responses by interacting with multiple partners to modulate transcription of downstream target genes. The transcription factor (TF) containing basic helix-loop-helix (bHLH) motif interacts physically with red and far-red photoreceptor, phytochrome, called Phytochrome Interacting Factors (PIF) [51]. The present study was also successful in identifying bHLH69 as conserved motif for PHYA gene (Fig. 3 and Table 2). The TF with bHLH motif has already been known to be involved in regulating circadian rhythm in Arabidopsis [52, 53]. Presence of DNA binding motif (bHLH) in PHYA indicates that it might compete with PIFs for DNA binding to repress flowering [51]. The present study also deciphered the domains encoded by exon 1 of LprPHYA3 (Fig. 4) which are in congruity with the Glycine max PHYA3 [3]. This implies to the fact that exon 1 in $\operatorname{LprPHYA3}$ codes for GAF and PAS domains with chances of conserved functions. The plant phytochromes detect light via their amino-terminal photosensory module (PSM) comprising N-terminal extension (NTE), period-ARNT-single-minded (PAS), cGMP-specific phosphodiesterase-adenylyl cyclase-FhlA (GAF) and phytochrome-specific (PHY) domains with the help of a bilin chromophore. C-terminal output module (OPM) is shared by two PAS on the $\mathrm{N}$-terminal side and a histidine kinase-related domain (HKRD) [54]. NTE is related to the stability of light-activated phytochromes and interacts with the part of GAF which binds to $P \Phi B$ for lyase activity and reversible Pr/Pfr photo-transformation. PAS domain represents transducer domain that mediates light signal from input photosensory domain to output module. HKRD domain plays major roles in dimerization, nuclear import and localization [55]. LprPHYA3-Exon 1 encodes GAF and PAS domain of phytochrome genes which belongs to the photo-sensory module and is responsible for convertible $\mathrm{Pr} / \mathrm{Pfr}$ transformation as well as light-signal transduction from this module to output module, respectively [56]. Characterization of full gene sequence of $P H Y A 3$ in Lablab purpureus would unravel the different domains involved in downstreaming lightmediated response to the signaling pathway along with their putative roles.

\section{Conclusion}

Partial characterization of LprPHYA3 would facilitate allelic characterization in relation to photoperiod responsive flowering in Lablab purpureus. Phylogenetic analysis indicated that complete characterization of LprPHYA3 would be possible utilizing sequence information from related legumes. The presence of conserved DNA binding motif (bHLH69) in PHYA gene indicated that it might repress flowering by competing for DNA binding with bHLH containing TFs. Domain analysis of protein-encoding $\operatorname{LprPHYA3}$ would unfold the signaling pathways and their interaction with different proteins from PEBP (Phosphatidyl ethanolamine-binding protein) family genes that would guide flowering response.

The continued progress in this direction would entice further questions to address in future like characterization and identification of allelic variants for LprPHYA3 and their role in modulating photoperiod responsive flowering. Additionally, qPCR studies could also be undertaken for relative expression studies of $P H Y A 3$ in LD and SD conditions. The role of LprPHYA3 may be confirmed through genome editing by utilizing partial sequence reported in the present study. These efforts would accelerate the understanding of flowering time and growth habit regulation in Lablab purpureus in response to changed photoperiod.

\section{Abbreviations}

R/FR: Red/Far-Red; PHY: Phytochrome; FT: Flowering locus T; LD: Long day; SD: Short day; DT: Determinate; IDT: Indeterminate; PS: Photosensitive; PIS: Photoinsensitive; SNP: Single nucleotide polymorphism; NCBI: National Centre 
for Biotechnology Information; BLAST: Basic Local Alignment Search Tool; MEGA: Molecular Evolution Genetics Analysis; MEME: (Multiple Em for Motif Elicitation); CTAB: Cetyl trimethyl ammonium bromide; PCR: Polymerase chain reaction; SMART: Simple Modular Architecture Research Tool; DNA: Deoxyribonucleic acid; PSM: Photosensory module; NTE: N-Phosphodiesterase-adenylyl cyclase-FhIA (GAF); PHY: Phytochrome-specific; OPM: Output module; HKRD: Histidine kinase-related domain; PIF: Phytochrome-interacting factors; CO: Constans; AP1: Apetela 1; TF: Transcription factors; bHLH: Basic helix-loophelix; PEBP: Phosphatidyl ethanolamine-binding protein

\section{Supplementary Information}

The online version contains supplementary material available at https://doi. org/10.1186/s43141-021-00295-z.

Additional file 1. Sequencing data.

\section{Acknowledgements}

The work was supported by the funds provided by Gujarat State Government, Gandhinagar, Gujarat, India, and Indian Council of Agricultural Research, New Delhi, India

\section{Authors' contributions}

SK recorded observations, did laboratory work and wrote the manuscript. KM conceptualized the research and analysed the data. VP guided laboratory works and interpreted the results. RP ensured funding for the laboratory work, helped in field evaluation and guided the research work. DC provided the experimental material, assisted in phenotyping and guided the research work. Further, all the authors have read and approved the manuscript.

\section{Funding}

This research work was supported by Department of Genetics and Plant Breeding, Navsari Agricultural University, Gujarat, India. The research fund was utilized for purchasing reagents and conducting laboratory works.

\section{Availability of data and materials}

The dataset supporting the conclusions of this article are included within the article. The sequencing data have been submitted as supplementary material.

\section{Declarations}

Ethics approval and consent to participate Not applicable.

\section{Consent for publication}

Not applicable.

\section{Competing interests}

The authors declare that they have no competing interests.

\section{Author details}

${ }^{1}$ Department of Genetics and Plant Breeding, N. M. College of Agriculture, Navsari Agricultural University, Navsari, Gujarat 396 450, India. ${ }^{2}$ Department of Basic Science and Humanities, ASPEE College of Horticulture and Forestry, NAU, Navsari, Gujarat 396 450, India. ${ }^{3}$ Pulses and Castor Research Station, Navsari Agricultural University, Navsari, Gujarat 396 450, India.

Received: 25 September 2021 Accepted: 28 December 2021 Published online: 13 January 2022

\section{References}

1. Xu M, Xu Z, Liu B, Kong F, Tsubokura Y, Watanabe S, Xia Z et al (2013) Genetic variation in four maturity genes affects photoperiod insensitivity and PHYA-regulated post-flowering responses of soybean. BMC Plant Biol 13:91-105. https://doi.org/10.1186/1471-2229-13-91
2. Sharrock RA, Mathews S (2006) Phytochrome genes in higher plants: structure, expression, and evolution. In: Photomorphogenes in plants and bacteria. Kluwer Academic Publishers, Dordrecht. https://doi.org/10. 1007/1-4020-3811-9_7

3. Watanabe S, Hideshima R, Zhengjun X, Tsubokura Y, Sato S, Nakamoto Y, Yamanaka N et al (2009) Map-based cloning of the gene associated with the soybean maturity locus E3. Genetics 182:1251-1262. https://doi.org/ 10.1534/Genetics.108.098772

4. Weller JL, Ortega R (2015) Genetic control of flowering time in legumes. Front Plant Sci. https://doi.org/10.3389/Fpls.2015.00207

5. Cronk Q, Ojeda I, Pennington RT (2006) Legume comparative genomics: progress in phylogenetics and phylogenomics. Curr Opin Plant Biol 9:99-103. https://doi.org/10.1016/j.Pbi.2006.01.01

6. Clack T, Mathews S, Sharrock RA (1994) The phytochrome apoprotein family in Arabidopsis is encoded by five genes: the sequences and expression of PHYD andPHYE. Plant Mol Biol 25:413-427. https://doi.org/ 10.1007/BF00043870

7. Sharrock RA, Quail PH (1989) Novel phytochrome sequences in Arabidopsis thaliana: structure, evolution, and differential expression of a plant regulatory photoreceptor family. Genes Dev 3:1745-1757. https://doi. org/10.1101/Gad.3.11.1745

8. Bernard RL (1971) Two major genes for time of flowering and maturity in soybeans 1. Crop Sci 11:242-244. https://doi.org/10.2135/Cropsci1971. $0011183 \times 001100020022 x$

9. Bonato ER, Vello NA (1999) E6, a dominant gene conditioning early flowering and maturity in soybeans. Genet Mol Biol 22:229-232. https:// doi.org/10.1590/S1415-47571999000200016

10. Buzzell RI (1971) Inheritance of a soybean flowering response to fluorescent daylength conditions. Can J Genet Cytol 13:703-707. https://doi. org/10.1139/G71-100

11. Buzzell RI, Voldeng HD (1980) Research notes: inheritance of insensitivity to long daylength. Soybean Genet Newsl 7:26-28

12. Cober ER, Voldeng HD (2001) A new soybean maturity and photoperiodsensitivity locus linked to E1 and T. Crop Sci 41:698-701. https://doi.org/ 10.2135/Cropsci2001.413698x

13. Cober ER, Molnar SJ, Charette M, Voldeng HD (2010) A new locus for early maturity in soybean. Crop Sci 50:524-527. https://doi.org/10.2135/Crops ci2009.04.0174

14. McBlain BA, Bernard RL, Cremeens CR, Korczak JF (1987) A procedure to identify genes affecting maturity using soybean isoline testers1. Crop Sci 27:1127-1132. https://doi.org/10.2135/Cropsci1987.0011183X0027000 $60008 x$

15. Xia Z, Watanabe S, Yamada T, Tsubokura Y, Nakashima H, Zhai H, Anai T et al (2012) Positional cloning and characterization reveal the molecular basis for soybean maturity locus E1 that regulates photoperiodic flowering. Proc Natl Acad Sci 109:E2155-E2164. https://doi.org/10.1073/Pnas. 1117982109

16. Franklin KA, Quail PH (2010) Phytochrome functions in Arabidopsis development. J Exp Bot 61:11-24. https://doi.org/10.1093/Jxb/Erp304

17. Liu B, Kanazawa A, Matsumura H, Takahashi R, Harada K, Abe J (2008) Genetic redundancy in soybean photoresponses associated with duplication of the phytochrome a gene. Genetics 180:995-1007. https://doi.org/ 10.1534/Genetics.108.092742

18. Cober ER, Tanner JW, Voldeng HD (1996) Soybean photoperiod-sensitivity loci respond differentially to light quality. Crop Sci 36:606-610. https:// doi.org/10.2135/Cropsci1996.0011183X003600030014x

19. Kwak M, Velasco D, Gepts P (2008) Mapping homologous sequences for determinacy and photoperiod sensitivity in common bean (Phaseolus Vulgaris). J Hered 99:283-291. https://doi.org/10.1093/Jhered/Esn005

20. Kong F, Liu B, Xia Z, Sato S, Kim BM, Watanabe S, Yamada T et al (2010) Two coordinately regulated homologs of Flowering Locus $T$ are involved in the control of photoperiodic flowering in soybean. Plant Physiol 154:1220-1231. https://doi.org/10.1104/Pp.110.160796

21. Maass BL, Knox MR, Venkatesha SC, Angessa TT, Ramme S, Pengelly BC (2010) Lablab purpureus - a crop lost for Africa? Trop Plant Biol 3:123-135. https://doi.org/10.1007/S12042-010-9046-1

22. Dhaliwal SK, Talukdar A, Gautam A, Sharma P, Sharma V, Kaushik P (2020) Developments and prospects in imperative underexploited vegetable 
legumes breeding: a review. Int J Mol Sci 21:9615. https://doi.org/10. 3390/ljms21249615

23. Modha K, Kale B, Borwal D, Ramtekey V, Arpit B (2019) Inheritance pattern of photoperiod responsive flowering, growth habit and flower colour in indian bean [Lablab Purpureus (L.) Sweet.]. Electron J Plant Breed 10:297. https://doi.org/10.5958/0975-928X.2019.00037.1

24. Ramtekey V, Bhuriya A, Ayer D, Parekh V, Modha K, Kale B, Vadodariya G et al (2019) Molecular tagging of photoperiod responsive flowering in indian bean [Lablab Purpureus (L.) Sweet]. Indian J Genet Plant Breed 79:264-269. https://doi.org/10.31742/JJGPB.79S.1.17

25. Kaldate S, Patel A, Modha K, Parekh V, Kale B, Vadodariya G, Patel R (2021) Allelic characterization and protein structure analysis reveals the involvement of splice site mutation for growth habit differences in Lablab purpureus (L.) sweet. J Genet Eng Biotechnol 19:34. https://doi.org/10 1186/S43141-021-00136-Z

26. Weller JL, Vander Schoor JK, Perez-Wright EC, Hecht V, González AM Capel C, Yuste-Lisbona FJ et al (2019) Parallel origins of photoperiod adaptation following dual domestications of common bean. J Exp Bot 70:1209-1219. https://doi.org/10.1093/Jxb/Ery455

27. Doyle JJ, Doyle JLA (1987) Rapid DNA isolation procedure for small quantities of fresh leaf tissue. Phytochem Bull 19:11-15

28. Hall TA (1999) A user-friendly biological sequence alignment editor and analysis program for windows 95/98/NT. Nucleic Acids Symp Ser 41:95-98

29. Altschul SF, Madden TL, Schäffer AA, Zhang J, Zhang Z, Miller W, Lipman DJ (1997) Gapped BLAST and PSI-BLAST: a new generation of protein database search programs. Nucleic Acids Res 25(17):3389-3402. https:// doi.org/10.1093/nar/25.17.3389

30. Kumar S, Stecher G, Li M, Knyaz C, Tamura K (2018) MEGA X: Molecular Evolutionary Genetics Analysis across computing platforms. Mol Biol Evol 35:1547-1549. https://doi.org/10.1093/Molbev/Msy096

31. Larkin MA, Blackshields G, Brown NP, Chenna R, McGettigan PA, McWilliam H, Higgins DG et al (2007) Clustal W and Clustal X version 2.0. Bioinformatics 23(21):2947-2948. https://doi.org/10.1093/bioinformatics/ btm404

32. Tamura K, Nei M (1993) Estimation of the number of nucleotide substitutions in the control region of mitochondrial DNA in humans and chimpanzees. Mol Biol Evol 10:512-526. https://doi.org/10.1093/oxfor djournals.molbev.a040023

33. Felsenstein (1985) Confidence limits on phylogenies: an approach using the bootstrap. Evolution 39(4):783-791. https://doi.org/10.1111/j.15585646.1985.tb00420.x

34. Bailey TL, Boden M, Buske FA, Frith M, Grant CE, Clementi L, Ren J, Li WW, Noble WS (2009) MEME SUITE: tools for motif discovery and searching. Nucleic Acids Res 37:W202-W208. https://doi.org/10.1093/nar/gkp335

35. Aman Beshir J, Kebede M (2021) In silico analysis of promoter regions and regulatory elements (motifs and CpG islands) of the genes encoding for alcohol production in Saccharomyces cerevisiaea S288C and Schizosaccharomyces pombe 972h. J Genet Eng Biotechnol 19:8. https://doi.org/10. 1186/s43141-020-00097-9

36. Lomsadze A, Ter-Hovhannisyan V, Chernoff YO, Borodovsky M (2005) Gene Identification in novel eukaryotic genomes by self-training algorithm. Nucleic Acids Res 33:6494-6506. https://doi.org/10.1093/Nar/ Gki937

37. Jones DT, Taylor WR, Thornton JM (1992) The rapid generation of mutation data matrices from protein sequences. Comput Appl Biosci 8:275-282. https://doi.org/10.1093/bioinformatics/8.3.275

38. Letunic I, Doerks T, Bork P (2009) SMART 6: recent updates and new developments. Nucleic Acids Res 37:D229-D232. https://doi.org/10.1093/ nar/gkn808

39. Harada K, Watanabe S, Xia Z, Tsubokura Y, Yamanaka N, Anai T, Krezhova D (2011) Positional cloning of the responsible genes for maturity loci E1, E2 and E3 in Soybean. In: Soybean—Genetics Nov. Tech. Yield Enhanc

40. Dhanasekar P, Reddy KS (2015) A novel mutation in TFL1 homolog affecting determinacy in cowpea (Vigna unguiculata). Mol Genet Genomics 290:55-65. https://doi.org/10.1007/S00438-014-0899-0

41. Kim SE, Okubo H (1995) Control of growth habit in determinate lablab bean (Lablab purpureus) by temperature and photoperiod. Sci Hortic 61(3-4):147-155

42. Tsubokura Y, Matsumura H, Xu M, Liu B, Nakashima H, Anai T, Kong F et al (2013) Genetic variation in soybean at the maturity locus E4 is involved in adaptation to long days at high latitudes. Agronomy 3:117-134. https:// doi.org/10.3390/Agronomy3010117

43. Weller JL, Batge SL, Smith JJ, Kerckhoffs LHJ, Sineshchekov VA, Murfet IC, Reid JB (2004) A dominant mutation in the pea PHYA gene confers enhanced responses to light and impairs the light-dependent degradation of Phytochrome A. Plant Physiol 135:2186-2195. https://doi.org/10 1104/Pp.103.036103

44. Zhang Y, Sun J, Xia H, Zhao C, Hou L, Wang B, Li A et al (2018) Characterization of peanut phytochromes and their possible regulating roles in early peanut pod development. PLoS One 13:5. https://doi.org/10.1371/ Journal.Pone.0198041

45. Hwang WJ, Ha J, Lee T, Jeong H, Kim MY, Kim SK, Lee Y-H et al (2017) A candidate flowering gene in mungbean is homologous to a soybean phytochrome a gene. Euphytica 213:79. https://doi.org/10.1007/ S10681-017-1866-8

46. Paterson AH, Lin Y-R, Li Z, Schertz KF, Doebley JF, Pinson SRM, Liu S-C et al (1995) Convergent domestication of cereal crops by independent mutations at corresponding genetic loci. Science 269:1714-1718. https://doi. org/10.1126/Science.269.5231.1714

47. Opperdoes (2003) Phylogenetic analysis using protein sequences. In: Phylogenetics Handb. A Pract. Approach to DNA Protein Phylogeny

48. McClean PE, Mamidi S, McConnell M, Chikara S, Lee R (2010) Synteny mapping between common bean and soybean reveals extensive blocks of shared loci. BMC Genomics 11:184. https://doi.org/10.1186/ 1471-2164-11-184

49. Chang Y, Liu H, Liu M, Liao X, Sahu SK, Fu Y, Song B et al (2019) The draft genomes of five agriculturally important African orphan crops. Gigascience 8:3. https://doi.org/10.1093/Gigascience/Giy152

50. Wang Z, Yang R, Devisetty UK, Maloof JN, Zuo Y, Li J, Shen Y et al (2017) The divergence of flowering time modulated by FT/TFL1 is independent to their interaction and binding activities. Front Plant Sci 8:697. https:// doi.org/10.3389/Fpls.2017.00697

51. Pham VN, Kathare PK, Huq E (2017) Phytochromes and phytochrome interacting factors. Plant Physiol 176:1025-1038. https://doi.org/10.1104/ pp.17.01384

52. Hanano S, Stracke R, Jakoby M, Merkle T, Domagalska MA, Weisshaar B, Davis SJ (2008) A systematic survey in Arabidopsis thaliana of transcription factors that modulate circadian parameters. BMC Genomics 9:182. https://doi.org/10.1186/1471-2164-9-182

53. Nakamichi $N$ (2011) Molecular mechanisms underlying the Arabidopsis circadian clock. Plant Cell Physiol 52(10):1709-1718. https://doi.org/10. 1093/pcp/pcr118

54. Oide M, Nakasako M (2021) Red light-induced structure changes in phytochrome A from Pisum sativum. Sci Rep 11:2827. https://doi.org/10. 1038/S41598-021-82544-2

55. Rockwell NC, Su Y-S, Lagarias JC (2006) Phytochrome structure and signaling mechanisms. Annu Rev Plant Biol 57:837-858. https://doi.org/ 10.1146/Annurev.Arplant.56.032604.144208

56. Cheng M-C, Kathare PK, Paik I, Huq E (2021) Phytochrome signaling networks. Annu Rev Plant Biol 72:217-244. https://doi.org/10.1146/Annur ev-Arplant-080620-024221

\section{Publisher's Note}

Springer Nature remains neutral with regard to jurisdictional claims in published maps and institutional affiliations. 